Espinal, L.; Sánchez, M.; Zapata, N. (2018). Cotización del petróleo y volumen de acciones de aerolíneas latinoamericanas 2011-2015: análisis de correlación bivariada. Contaduría Universidad de Antioquia, 73, 49-78.

Doi: https://doi.org/10.17533/udea.rc.n73a03

\title{
Cotización del petróleo y volumen de acciones de aerolíneas latinoamericanas 2011-2015: análisis de correlación bivariada*
}

\author{
Leidy Johana Espinal Zapata \\ leidyespinal14@gmail.com \\ Cooperativa de Profesores Universidad de Antioquia
}

Moisés Sánchez Toro

moisess.toro@gmail.com

Grupo Bancolombia S.A.

Natalia Zapata Ruiz nataliazapataruiz@gmail.com

San Guzney S.A.S.

Este artículo, elaborado en 2016, es el resultado del proyecto de Investigación financiado por el Centro de Investigaciones y Consultorías (CIC) de la Facultad de Ciencias Económicas de la Universidad de Antioquia en la Convocatoria para apoyar proyectos de investigación de los estudiantes de pregrado de la Facultad de Ciencias Económicas. Acta No 21502401-05-2016 (Acta de Inicio y aceptación del Proyecto No. CT119 del 18 de abril de 2016). 
Cotización del petróleo y volumen de acciones de aerolíneas latinoamericanas 2011-2015: análisis de correlación bivariada

Resumen: El presente artículo tiene como objetivo analizar la influencia del precio del petróleo en el costo y volumen de las acciones de aerolineas latinoamericanas en el periodo 2011-2015; para lograr esto, después de realizar una revisión de la literatura más relevante sobre el tema, analizar el comportamiento de las acciones de seis aerolíneas (Volaris, LAN, Gol, Copa, Avianca y Aeroméxico) y el precio del petróleo en las referencias BRENT y WTI, se aplicó un análisis de correlación bivariada. Según los análisis se concluye que la fluctuación del precio del petróleo, una de las principales materias primas en la aviación, afecta de manera negativa el precio y volumen de las acciones y repercute en la economía y finanzas de las aerolíneas y el desempeño del sector. Este estudio tiene implicaciones para las aerolíneas, los analistas y académicos que siguen el comportamiento de estas compañias.

Palabras clave: Valoración de empresas, Aerolíneas Latinoamericanas, Petróleo, Múltiplos Comparables de Mercado, Análisis de correlación bivariada.

Oil prices and share volume of Latin American airlines 2011-2015: bivariate correlation analysis.

Abstract: The present paper has the aim of analyzing the influence of oil price on the cost and volume of the shares of Latin American Airlines in the period 2011-2015. To achieve this aim, a review of the most relevant literature on the topic was first conducted, and then share behavior of six airlines, (Volaris, LAN, Gol, Copa, Avianca and Aeroméxico) and oil price in the BRENT and WTI references were analyzed, and finally a bivariate correlation analysis was applied. According to the analyses, it is concluded that price fluctuation of oil, one of the main inputs in aviation, negatively affects share price and volume and impacts on the economy and finances of the airlines and on the performance of the sector. This study has implications for airlines, analysts, and scholars who follow the behavior of these companies.

Keywords: Firm valuation, Latin American airlines, Oil, Comparable Market Multiples, Bivariate correlation analysis.

Le cours du pétrole et le volumen d'actions des compagnies aériennes latino-américaines 2011-2015: une analyse de corrélation bivariée.

Résumé: cet article a pour but d'analyser l'influence du prix du pétrole sur le coût et sur le volume des actions des compagnies aériennes latino-américaines pour la période comprise entre 2011 et 2015. Pour ce faire, après avoir révisé la littérature la plus significative sur ce sujet; après avoir analysé le comportement des actions de six compagnies aériennes (Volaris, LAN, Gol, Copa, Avianca et Aeroméxico) ainsi que le prix du pétrole dans les références BRENT et WITI; nous avons mené une analyse de corrélation bivariée. D'après ces analyses, nous avons pu conclure que la fluctuation du prix du pétrole, une des matières premières dans l'aviation, touche de manière négative le prix et le volumen des actions. Ceci a une répercussion sur l'économie et les finances des compagnies aériennes et donc sur la performance du secteur. Cette étude concerne non seulement les compagnies aériennes mais aussi les analystes et académiciens qui surveillent le comportement de ce genre de compagnies.

Mots-Clés: valorisation des entreprises, compagnies aériennes latinoaméricaines, pétrole, multiples comparables du marché, analyse de corrélation bivariée.

Cotização do petróleo e volume de ações das Linhas Aéreas latino-americanas 2011-2015: Análise de Correlação bivariada

Resumo: Este artigo tem como objetivo analisar a influência do preço do petróleo no custo e volume das ações de linhas aéreas latino-americanas no periodo 2011-2015; para atingir este objetivo, após realizar uma revisão da literatura mais relevante sobre o assunto, analisar o comportamento das ações de seis linhas aéreas (Volaris, LAN, Gol, Copa, Avianca e Aeroméxico) e o preço do petróleo nas referências BRENT e WTI, se fez uma análise de correlação bivariada. Segundo as reflexões, conclui-se que a flutuação do preço do petróleo, uma das principais matérias-primas na aviação, afeta negativamente o preço e o volume das ações e repercute na economia e finanças das linhas aéreas e o desenvolvimento do setor. Este estudo tem implicações para as linhas aéreas, os analistas e acadêmicos que seguem o comportamento destas empresas.

Palavras Chave: Valoração de empresas, Linhas aéreas latino-americanas, Petróleo, avaliação por múltiplos, análise de correlação bivariada 
Cont. udea (julio-diciembre), pp. 49-78. (C) Universidad de Antioquia-2018.

\title{
Cotización del petróleo y volumen de acciones de aerolíneas latinoamericanas 2011-2015: análisis de correlación bivariada
}

\author{
Leidy Johana Espinal Zapata, Moisés Sánchez Toro, Natalia Zapata Ruiz \\ Doi: https://doi.org/10.17533/udea.rc.n73a03
}

Primera versión recibida en septiembre 2018 - Versión final aceptada en octubre 2018

\section{Introducción}

I as diferentes crisis del petróleo al igual que las variaciones constantes en Loferta y demanda han sido las causantes de las oscilaciones de los precios finales al consumidor. El comportamiento del petróleo a partir del 2011, según Cárdenas (2015) y Mateo y García (2014), ha sido muy fluctuante, por las especulaciones que se generan alrededor de esta materia prima.

En la revisión de la literatura se analiza que sobre el petróleo e impactos económicos se tiene una completa biografía del tema; autores como Barsky y Kilian (2004) destacan de manera parcial dicha situación. Sin embargo, pocos son los estudios e investigaciones enfocados en los efectos directos que generan las variaciones de los precios de petróleo sobre las empresas prestadoras de servicio aéreo en Latinoamérica.

Las aerolíneas en Latinoamérica específicamente, según Graham, Kaplan y Sibley (1983) y Forgas et al. (2011), han tomado gran impulso en desarrollo, crecimiento y participación de mercado desde 1970, gracias a la desregulación paulatina del sector (Bisignani, 2006), a políticas gubernamentales y a la conciencia progresiva de clientes de utilizar el servicio con mayor frecuencia.

En este sentido, es evidente que bajo este contexto del comportamiento del precio del petróleo, existen consecuencias para el sector, considerando, además, que este tiene gran participación en la economía mundial: según un informe publicado por (ATAG, 2017) las aerolíneas transportaron anualmente más de 4.000 millones de pasajeros, se generaron un total de 65,5 millones de empleos a nivel mundial y aportó 2,7 billones a la economía mundial. 
Espinal, L.; Sánchez, M.; Zapata, N. Cotización del petróleo y volumen de acciones de aerolíneas...

Asimismo, las aerolíneas latinoamericanas son una parte importante en la economía mundial que cuenta con "15.000 aviones de servicio, aproximadamente 10.000 aeropuertos, operaciones a través de rutas de 15 millones de $\mathrm{km}$ de longitud, un tercio de las exportaciones de productos manufacturados fueron transportados vía aérea, la industria genera 32 millones de empleos y aporta alrededor del $8 \%$ al PIB mundial" (Capoccitti, Khare, y Mildenberger, 2010, p. 67); donde "las participaciones en los costos de combustible tienden a ser mayores con equipos más antiguos, con tamaño de las flotas más pequeñas" (Meland, 2014, p. 40).

Se ha identificado un problema y es que, si la mayor parte de la estructura de costos de las aerolíneas está sujeta al precio del combustible, cualquier variación que se produzca en este, afectará de manera directa dicha estructura, "los combustibles en la aviación representan una tercera parte de los costos de operación" (Calderón, 2012, p. 21); en este sentido, es predecible un cambio en el valor organizacional derivado de los movimientos en los costos.

A partir de la principal premisa de las finanzas donde la función de una empresa es crear valor, aparece la valoración de empresas como estrategia empresarial, según lo señalan Moscoso y Botero (2013). La valoración constituye la forma como se concibe la empresa en su conjunto, a través de aspectos de carácter cualitativo y cuantitativo como lo es la estructura de costos descrita anteriormente. Así pues, entra a jugar un papel fundamental el precio de la compañía en la valoración, concibiendo éste como un inductor de valor, más no el valor per se, puesto que este último es el resultado de la percepción y el diagnóstico que se haya hecho previo; "el precio es un hecho definido, que se da al momento de una negociación” (Montero, 2011, p. 32).

Se tiene como objetivo analizar la influencia del precio del petróleo en el precio y volumen de las acciones de aerolíneas latinoamericanas en el periodo 2011-2015. Para esto, se evaluó el comportamiento de las acciones de seis aerolíneas y el precio del petróleo para establecer relaciones entre ambas variables. Como valor agregado, se determinó el comportamiento y evolución de múltiplos de mercado de estas empresas.

La metodología utilizada se dio mediante un modelo de correlación bivariada que contrasta tres variables: precio del petróleo con dos de sus referencias (WTI y BRENT), precio y volumen de las acciones de aerolíneas. La muestra considerada comprende seis aerolíneas latinoamericanas: Copa Airlines, Grupo Aeroméxico, Volaris, Avianca, Gol Aerolíneas Inteligentes y LAN, basados en su tamaño y cotización en bolsa. Se obtuvieron datos diarios tanto de la cotización de las acciones como del precio del petróleo en un horizonte de tiempo desde 2011 hasta 2015.

Se encontró que las fluctuaciones del precio del petróleo impactan el precio y volumen de las acciones de las aerolíneas y a su vez afectan su valor 
económico; el precio de las acciones es un inductor de valor y más aún cuando se analiza los múltiplos de las aerolíneas, los cuales no se pueden analizar de manera aislada, puesto que cada uno tiene en cuenta variedad de factores que revelan particularidades de las empresas.

Además de la presente introducción, este documento se compone de la siguiente manera, en la segunda sección se realiza una aproximación a la literatura existente con el fin de formular las hipótesis utilizadas en la investigación; en la tercera sección se expone los principales hallazgos; y se concluye con las consideraciones finales con el ánimo de evidenciar el cumplimiento de las hipótesis formuladas.

\section{Revisión de literatura}

\section{II.1 Panorama Actual del Sector}

El contexto económico de Latinoamérica, los ciclos económicos a los cuales hace referencia Llinás (2002) y el sector de servicios de transporte aéreo, como lo mencionan los autores González y Hernández (2016), Perilla (2009) y Perilla (2010) se han visto afectados desde la década de 1990 por el comportamiento fluctuante que ha tenido el precio del petróleo, por la inmersión de aerolíneas de bajo costo en el mercado, por la desregularización del sector (Valdés y Ramírez, 2011) y por los costos de operación, según Ginieis, Sánchez y Campa (2012).

Aunque aparenta ser un panorama poco alentador, el fuerte crecimiento económico y el comercio internacional que experimentó la región latinoamericana en el año 2011, según la International Air Transport Association (IATA), en 2014 impulsó el transporte aéreo de pasajeros y de carga e incrementó los beneficios de las aerolíneas de la región, a pesar de que el encarecimiento del petróleo frena el crecimiento económico mundial (Gutiérrez, 2015).

Es pertinente mencionar la mayor depresión aérea en la década del setenta. Según Helmbold (2004) y González y Mesa (2007), con el elevado precio del petróleo la estructura de gastos no se pudo sobrellevar y de un modo u otro fueron los gobiernos los que tuvieron que afrontar la situación por las compañías aéreas. Durante la crisis de 1972, Southwest Airlines prefirió vender cuatro aviones de la flota en lugar de despedir empleados (Zapata, 2012). Esto demuestra la exposición existente con relación al precio del oro negro.

Esta exposición se puede ver intensificada con la propagación de la noticia de variaciones significativas en el precio del petróleo. Es decir, cuando el crudo está a un precio que definitivamente genera impactos sobresalientes, y además es anunciado de forma masiva, se provoca un pánico generalizado en el mercado alterando precios accionarios debido a que cuando existe miedo los 
Espinal, L.; Sánchez, M.; Zapata, N. Cotización del petróleo y volumen de acciones de aerolíneas...

individuos actúan con más decisión para proteger su capital. Algo relacionado se ha identificado en estudios de precios accionarios cuando ocurren anuncios sobre fallos en la cadena de suministro en el rendimiento de las acciones

The average abnormal stock returns of firms that experienced disruptions is nearly $-40 \%$. Much of this underperformance is observed in the year before the announcement, the day of the announcement, and the year after the announcement. Furthermore, the evidence indicates that firms do not quickly recover from the negative effects of disruptions. (K. Hendricks, 2005)

Un estudio similar sobre el tema, pero con el foco puesto en la riqueza de los accionistas demuestra el impacto definitivo en el decrecimiento del precio de las acciones "The evidence clearly shows that on average, announcements of supply chain glitches are associated with severe stock price decreases. The stock market reaction is negative on both days of the event period" (K. B. Hendricks y Singhal, 2003)and firms with higher growth prospects experience a more negative reaction. There is no difference between the stock market's reaction to pre-1995 and post-1995 glitches, suggesting that the market has always viewed glitches unfavorably. Capital structure (debt-equity ratio.

Los accionistas de las compañías de aerolíneas son directamente afectados por cualquier circunstancia que provoque una variación en el precio de las acciones de las compañías. Así que la especulación sobre los precios del combustible traerá consecuencias poco deseables sobre dichos actores y necesariamente habrá que idear estrategias para evitar una crisis severa. Cualquier acción que los accionistas definan tomar, tendrá repercusiones en el valor y por lo tanto en el precio de las acciones.

El sector de servicios de transporte aéreo es bastante vulnerable a la fluctuación de los precios del combustible (Helmbold Gretel, 2004) y (Moreno y Sandoval, 2011). Dado esto, según (Cano, 2015, p.41) "una alineación entre un ciclo económico malo y una alta volatilidad en los precios del petróleo podría ser fatal para la industria". Esta vulnerabilidad se presenta porque solo se cuenta con combustibles fósiles como fuente de energía primordial ya que los biocombustibles se encuentran en período de desarrollo (Criollo, 2013). Incluso alzas importantes en los precios del petróleo pueden producir fenómenos societarios, ya que pueden acelerar los procesos de fusión en el sector, entre las aerolíneas para buscar socios y reducir costos (Zapién, 2010).

El combustible de aviación representa cerca de un $40 \%$ de los costos de las empresas aéreas (Moreno y Sandoval, 2011). En Colombia, el combustible constituye el $35 \%$ de los costos operacionales de las aerolíneas de pasajeros y el $39 \%$ para las aerolíneas de carga (González y Muñoz, 2014). Los combustibles en la aviación influyen de forma directa en la parte económica de este sector, ya que representa una tercera parte de los costos de operación (Criollo, 2013). 
Conforme lo señalan Agostini (2008) y Valdés (2009), en la desregulación del sector de servicios de transporte aéreo de Latinoamérica en los últimos años han intervenido con mayor fuerza variables económicas y políticas que lo favorecen. Dentro de este horizonte es preciso señalar que a menos barreras relacionadas con normatividad (Cuevas, 2009) y mayores ayudas del gobierno, son más las aerolíneas de bajo costo que se han impulsado y posicionado en el mercado.

Aunque ésta sea la realidad para el sector, la constante creación e inmersión de aerolíneas de bajo costo, es un factor preocupante por la competencia de mercado que se genera (Candela, 2008), no obstante, Gönenç y Nicoletti (2001) y Armstrong y Sappington (2006) hablan sobre servicios adicionales como mayor valor agregado.

Esta nueva línea de mercado de bajo costo está muy relacionada tanto con la desregulación del sector como a los cambios fluctuantes y comportamientos del petróleo en los últimos años (Gutiérrez, 2016) y para dar respuesta a esta hipótesis es indispensable conocer la historia, como lo mencionan Cárdenas (2015) y Barsky y Kilian (2004).

\section{II.2 Volumen y precio de las acciones dentro del valor de las empresas}

En la mayoría de los mercados existe una causalidad de la rentabilidad hacia el volumen, lo cual implica que mayores variaciones de precios trae consigo mayores variaciones de volumen (Rojas y Kristjanpoller, 2015). Pero el volumen no debe ser visto sólo como una medida derivada de las transacciones. El volumen juega un papel más allá de ser un parámetro descriptivo del proceso de negociación (Blume, Easley, y O’Hara, 1994).

El volumen es visto como un indicador. Es uno de los factores que señala el desarrollo del mercado de capital de un país, según señalan Pagano, Panetta, y Zingales (1998) y Borges, Yen-Tsang y Maciel (2011).

\section{II.3 Generalidades de la valoración de empresas}

La principal responsabilidad de la administración de una empresa es maximizar su valor (Álvarez, García, y Borraez, 2006). Según autores como Blanco (2009) y Santandreu y Torres (2012), el permanente empeño por crear valor ha llevado a que uno de los principales temas de investigación en finanzas sea la valoración de empresas, pues más que tendencia empresarial, es oportuno considerarlo como estrategia de mercado, según Moscoso y Botero (2013), además, el entorno competitivo en el contexto de la globalización, "junto al aumento del número de fusiones, adquisiciones y al interés de los acreedores, accionistas y nuevos inversores, llevarán a los propietarios a cuestionarse el valor de sus empresas" (Ribeiro y Anson, 2007, p. 124). 
Espinal, L.; Sánchez, M.; Zapata, N. Cotización del petróleo y volumen de acciones de aerolíneas...

Como lo señalan Blanco (2009) y Fernández (2008), el proceso de valoración es fundamental en tanto se ha convertido en una herramienta necesaria para tomar decisiones que implica definir el atractivo de un negocio. La valoración constituye la forma como se concibe la empresa en su conjunto, por esto, aspectos cualitativos y cuantitativos deben ser tenidos en cuenta. Narváez (2009) y Santandreu y Torres (2012) indican que este proceso empieza con el análisis del sector y la empresa, evaluación de los proveedores, clientes, competidores actuales y futuros, fortalezas, amenazas y necesidades de la compañía para poder realizar proyecciones de su valor.

Existe una gran diferencia entre el valor y el precio de una empresa. Fernández (2008) señala la importancia de no confundir el precio de la transacción con el valor de la empresa puesto que este último es el resultado de la percepción y el diagnóstico previo que se haya hecho; "el valor es un proceso técnico [...], en cambio, el precio es un hecho definido, que se da al momento de una negociación” (Montero, 2011, p. 32). Por otro lado, Serrano (2000), Realp (2009) y Montero (2011) agregan que pueden existir diferentes valores para un mismo activo debido a factores subjetivos, poder de negociación, percepción sobre expectativas futuras, entre otras.

Serrano (2000), Álvarez, García y Borraez (2006), Ribeiro y Anson (2007), Realp (2009) y Moscoso y Botero (2013), convergen en la premisa de que toda valoración además de considerar factores objetivos, implica estimaciones subjetivas, por tanto, es muy posible que una empresa nunca tenga un único valor definitivo.

Existen diferentes modelos en la valoración de compañías, el más común dentro de la literatura consultada es el modelo de descuentos de flujos de caja, Fernández (2008) lo describe como un método conceptual correcto, pues concibe a la empresa como un ente generador de flujos, éste modelo también es abordado por otros autores como Serrano (2000), Sarmiento y Cayón (2004), Álvarez et al (2006) Ribeiro y Anson (2007), Blanco (2009), Realp (2009), Montero (2011), Santandreu y Torres (2012) y Moscoso y Botero (2013) ${ }^{1}$. Aunque este método es el más aceptado, toda la literatura consultada hace mención a los múltiplos comparables de mercado, autores como Ribeiro y Anson (2007), Fernández (2008), los incluyen dentro de los métodos basados en la cuenta de resultados; por el contrario, Badenes y Santos (1999),

1 Se recomienda al lector consultar los diferentes modelos abordados por los autores mencionados, en el artículo no se hace una descripción de cada uno, puesto que no es del alcance del mismo. El lector podrá encontrar que autores como Fernández (2008) clasifican los modelos de valoración en métodos basados en el balance, métodos basados en las cuentas de resultados, métodos mixtos, métodos basados en el descuento de flujos de fondos, métodos basados en la creación de valor y métodos basados en opciones. 
Realp (2009), Santandreu y Torres (2012) los denominan de manera independiente como múltiplos comparables;

Realp (2009, p. 204) establece que la metodología de los múltiplos comparables de mercado "consiste en la obtención de una serie de ratios a partir de la capitalización bursátil o del precio pagado en transacciones de mercado de compañías similares, y su posterior aplicación sobre las magnitudes de la sociedad que es objeto de valoración". Álvarez et al. (2006) indican que se deben aplicar tres pasos básicos en esta metodología: i) elaborar un indicador de valoración para comparar el valor de la empresa con el atributo estratégico ${ }^{2}$ escogido; ii) analizar la evidencia existente y establecer un nivel estándar para el indicador y iii) la valoración se realiza, según el indicador al atributo estratégico.

\section{II.4 Múltiplos de operaciones realizadas en el sector transporte}

Fernández (2013), lista los siguientes múltiplos para valorar las empresas de transporte:

Price-to-Earnings ratio (PER): Relación precio - ganancia; es quizás uno de los múltiplos a los que más se hace alusión en la literatura consultada ${ }^{3}$ Badens y Santos (1999), Álvarez et al. (2006), Santandreu y Torres (2012) lo señalan como uno de los más utilizados. Álvarez et al. (2006, p. 68) menciona que "Permite conocer la capacidad actual de generación de excedentes de la empresa", es decir, el valor por acción sobre la utilidad por acción.

Precio/Ventas ( $\mathrm{P} /$ Ventas): mencionado también por autores como Badens y Santos (1999), Sarmiento y Cayón (2004), Álvarez et al. (2006), Fernández (2008) y Moscoso y Botero (2013), mide cuánto están dispuestos a pagar los inversores por cada peso de ventas.

P/EBITDA ${ }^{4}$ : mencionado también por autores como Sarmiento y Cayón (2004) y Álvarez et al. (2006).

P/EBIT's: mencionado también por Sarmiento y Cayón (2004).

2 Las operaciones de compra y venta de compañías en muchos casos están motivadas por factores que van mucho más allá de los factores económicos o financieros de la empresa. Los llamados indicadores estratégicos intentan captar este aspecto, proponiendo multiplicadores para determinar el precio de una empresa, donde estos multiplicadores son generados a partir de la experiencia de los mercados. (Álvarez et al., 2006, p. 67)

3 Se recomienda consultar a Sarmiento y Cayón (2004), b

4 Price/Earnings before interest, taxes, depretiation and amortization, "se le considera una medida de rentabilidad y por lo tanto un indicador que permite aproximarse al valor de una empresa" (Bastidas, 2007, p. 42).

5 Price/Earnings Before Interest and Taxes: Mide el beneficio que una empresa genera de sus operaciones 
Espinal, L.; Sánchez, M.; Zapata, N. Cotización del petróleo y volumen de acciones de aerolíneas...

Otros múltiplos utilizados en la práctica son: EV/EBITDA ${ }^{6}$, EV/VENTAS ${ }^{7}, \mathrm{P} /$ $\mathrm{FCL}^{8}, \mathrm{P} / \mathrm{VL}^{9}$ utilizado en el estudio realizado por Correa, Martínez, Ruiz y Yepes (2018).

Con base en la revisión de literatura anterior, se definen las siguientes hipótesis:

H1: Las fluctuaciones del precio del petróleo impactan el precio y volumen de las acciones de las aerolíneas, es decir, afectan su valor.

H2: Una cotización baja del petróleo incrementa la rentabilidad y liquidez de las aerolíneas y por lo tanto se fomenta al crecimiento de la industria y de la economía.

Diseño metodológico

La metodología ${ }^{10}$ utilizada es un análisis de correlación bivariada, consistente en estudiar la relación lineal entre tres variables aleatorias cuantitativas: el precio del petróleo (con dos de sus referencias: WTI y BRENT), el precio de la acción y el volumen de acciones de las aerolíneas. Además, se utilizaron ocho indicadores: PER, P/Ventas, P/EBITDA, P/EBIT, EV/EBITDA, EV/ Ventas, P/FCL, PER, P/VL.

Las Aerolíneas Copa Airlines, Grupo Aeroméxico, Volaris, Avianca, Gol Aerolíneas Inteligentes y LATAM (LAN) estaban activas en el momento del estudio; para seleccionarlas se tuvieron en cuenta dos factores clave a la hora de acceder a información, el tamaño de la aerolínea y su cotización en la bolsa local. Primero se consultaron las aerolíneas por países: Argentina, Brasil, Chile, Colombia, Costa Rica, Cuba, Ecuador, El Salvador, Guatemala, México, Panamá, Paraguay, Perú, República Dominicana y Venezuela. En países como Costa Rica, Cuba, Ecuador, El Salvador, Guatemala, Paraguay, República Dominicana y Venezuela, el tamaño de las aerolíneas no se consideró representativo para tomarlas dentro de la muestra, además, el acceso a información corporativa era mínimo o nulo en su totalidad. Por otra parte, en países como Perú opera Avianca Perú, la cual tiene su casa matriz en Colombia, por tanto, ya estaba considerada dentro de la muestra. Tras este filtro quedaron las empresas que se relacionan en la tabla 1 :

6 Enterprise Value/EBITDA: Determina si la empresa incorpora valor en mayor o menor cuantía que el directamente relacionado con los recursos generados y, si la gestión de la empresa es superior o inferior a los resultados obtenidos.

7 Enterprise Value/Sales: Esta ratio interpreta cuántas veces el valor de la empresa supera las ventas anuales.

8 Price to Free Cash Flow: Relaciona la capitalización bursátil de una empresa de acuerdo a los flujos de caja libre.

9 Precio/Valor en Libros: Compara el valor de una empresa con su valor contable o en libros.

10 Los autores suministraran la base de datos construida y utilizada en el presente artículo a cualquier investigador que la requiera mediante solicitud al Correo: articulo2016@gmail.com. 
Por último, se excluyeron de la muestra a Aerolíneas Argentinas, Air Panamá y Mexicana Airlines puesto que no cotizan en bolsa y el acceso a información es muy restringido, además, esta última se declaró en quiebra.

Tabla 1. Aerolíneas seleccionadas

\begin{tabular}{lccc}
\hline \multicolumn{1}{c}{ Aerolínea } & Ubicación geográfica & Cotización en bolsa & Flota \\
\hline Volaris & México & $\mathrm{Si}$ & 112 \\
Lan & Chile & $\mathrm{Si}$ & 331 \\
Gol & Brasil & $\mathrm{Si}$ & 144 \\
Copa Airlines & Panamá & $\mathrm{Si}$ & 100 \\
Avianca & Colombia & $\mathrm{Si}$ & 191 \\
Grupo Aromexico & México & $\mathrm{Si}$ & 134 \\
Aerolíneas argentinas & Argentina & No & 77 \\
Air panamá & Panamá & No & 17 \\
Mexicana Airlines & México & No & 130 \\
\hline
\end{tabular}

Fuente: elaboración propia con base en información tomada de Bloomberg.

Las bases teóricas en las que se fundamenta este paper son los costos del sector de servicios de transporte aéreo y el precio de la acción como inductor de valor. El modelo para el estudio es estimado según la función de autocorrelación:

Donde:

$$
R_{(k)}=\frac{E\left[\left(X_{i}-\mu_{\mathrm{x}}\right)\left(Y_{i}-\mu_{y}\right)\right]}{\sigma_{x} \sigma_{y}}
$$

$R_{(k)}$ coeficiente de correlación.

$X_{i} \quad$ precio de la acción de la empresa.

$\mu_{\mathrm{x}} \quad$ media del precio de la acción de la empresa.

$Y_{i} \quad$ precio de la referencia del petróleo.

$\mu_{y} \quad$ media del precio del petróleo.

$\sigma_{x}$ desviación estándar del precio de la acción de la empresa.

$\sigma_{y}$ desviación estándar del precio del petróleo.

Para seleccionar la información considerada en las variables se tomaron datos históricos del precio y volumen de las acciones de las aerolíneas de la muestra y datos históricos del precio del petróleo (referencias WTI y BRENT).

Los datos recopilados corresponden a cotizaciones diarias del precio desde 2011, para las dos referencias de petróleo, las aerolíneas Aeroméxico, Avianca, Copa Airlines, Gol Aerolíneas Inteligentes y LAN, y 2013 para Volaris; obtenidos de Bloomberg. 
Espinal, L.; Sánchez, M.; Zapata, N. Cotización del petróleo y volumen de acciones de aerolíneas...

Para el precio del petróleo se tuvo en cuenta un intervalo de tiempo que considerara periodos donde se evidenciara su fluctuación, tanto en momentos donde estuvo en auge como en los que se presentaron fuertes caídas en su cotización, en cuanto al precio de las acciones se encontró información disponible a partir de 2011, excepto para Volaris que empezó a cotizar en bolsa en 2013.

\section{Resultados}

A continuación, se muestran algunas variables financieras a partir de las cuales se identificaron hallazgos significativos.

Tabla 2. Información financiera aerolíneas

\begin{tabular}{lcccccc}
\hline \multicolumn{1}{c}{ Concepto } & Aeroméxico & Avianca & Copa & Gol & Lan & Volaris \\
\hline Capitalización de Mercado & 1,670 & 532 & 2,025 & 220 & 2,864 & 1,736 \\
Valor Empresa & 2,498 & 3,539 & 2,641 & 2,216 & 10,566 & 1,528 \\
Capital & 1,817 & 4,846 & 2,888 & 1,258 & 11,964 & 488 \\
Ventas & 2,733 & 4,361 & 2,250 & 2,980 & 9,740 & 1,148 \\
EBITDA & 323 & 450 & 401 & 73 & 1,383 & 187 \\
Ingresos Totales & 2,733 & 4,361 & 2,250 & 2,980 & 9,740 & 1,148 \\
Efectivo y equivalentes & 361 & 485 & 205 & 410 & 1,405 & 299 \\
Activos & 2,905 & 6,588 & 3,715 & 2,618 & 18,101 & 886 \\
Pasivo & 2,277 & 5,216 & 2,128 & 3,709 & 15,164 & 490 \\
Patrimonio & 627 & 1,373 & 1,587 & $(1,091)$ & 2,938 & 396 \\
\hline
\end{tabular}

(“) Cifras expresadas en millones de USD a Diciembre 31 de 2015

Fuente: elaboración propia con base en información tomada de Bloomberg.

A través de la capitalización de mercado se puede determinar el tamaño de una empresa. Cabe destacar que en el campo financiero todas están en el rango de pequeñas capitalizaciones, las cuales están comprendidas entre 250 y 2,000 millones de dólares. Sin embargo, se observan dos excepciones, Copa y LAN que exceden los 2,000 millones, por lo tanto, pasarían a ser capitalizaciones medias, ya que estas van hasta los 10,000 millones de dólares. La otra particularidad es, la situación de Gol a causa de que es considerada como micro capitalización porque está entre 50 y 250 millones. Esto es importante porque el estudio abarca tres niveles considerables de capitalización de mercado.

El valor de la empresa involucrado acá es uno sugerido por Bloomberg, que consiste en que el valor de la empresa es igual a la capitalización de mercado - efectivo y equivalentes + acciones preferentes + interés minoritario + deuda 
total. En este aspecto LAN es la compañía líder, pero algo notable es Avianca, ya que, a pesar de tener una capitalización media, es la segunda compañía más valiosa.

Con relación al aporte de los socios las aerolíneas dirigentes son las mismas que las del valor, es decir, LAN y Avianca. Esto quiere decir que hay mucho más compromiso financiero, ya que los montos son más considerables. Por diferencias significativas sobresalen de entre las demás, convirtiéndolas de nuevo en líderes aéreas para Latinoamérica.

En relación a la tabla 2, a continuación, se discrimina según las ventas el \% de EBITDA de las aerolíneas y, es evidente que se manejan rangos importantes a excepción de Gol, esto quiere decir, que el desempeño operativo no es muy fuerte porque esta medición enfocada en la generación de caja es desalentadora, ya que las compañías competentes exceden el $10 \%$.

\begin{tabular}{lc}
\hline \multicolumn{1}{c}{ Empresa } & \% EBITDA \\
\hline Aeroméxico & 11,81 \\
Avianca & 10,31 \\
Copa & 17,82 \\
Gol & 2,45 \\
LAN & 14,20 \\
Volaris & 16,32 \\
\hline
\end{tabular}

Fuente: elaboración propia con base en información tomada de Bloomberg.

Ahora, con base en el ingreso total, Gol tiene buenos datos, aunque en el párrafo anterior se observan las limitantes para sincronizar estos con la caja y como es sabido, ésta es la más representativa en las organizaciones. Volaris es la más débil en este aspecto, ya que se encuentra en el nivel más bajo con $\$ 1,147,899,600$ y es la única que no alcanza por lo menos los 2 mil millones de dólares.

Según la relación de pasivos y patrimonio con respecto a los activos, se observa que Volaris cuenta con un porcentaje de 55,28\% y Copa con 57,28 \%, es decir, estas empresas están muy equilibrados ya que respaldan sus activos con deuda mientras que el resto de las aerolíneas lo hacen con patrimonio, esto es un buen síntoma ya que no hay un riesgo latente de propiedad. Las demás compañías obtienen un promedio de financiación por parte de pasivos del $95,76 \%$, un dato bastante alto que a la vez es alterado por Gol que tiene una situación particular porque cuenta con $141,69 \%$ de financiación de pasivos ya que ha incurrido en gran déficit patrimonial, es decir, tiene un patrimonio de $-1,091,304,800$, esto es lógico siempre y cuando la compañía presenta pérdidas 
Espinal, L.; Sánchez, M.; Zapata, N. Cotización del petróleo y volumen de acciones de aerolíneas...

o producto de las pérdidas acumuladas en uno o varios ejercicios. Aeroméxico, Avianca y LAN presentan un riesgo de propiedad, ya que sus cifras de pasivos con relación a los activos son respectivamente $78,41 \%, 79,17 \%, 83,77 \%$, así que se nota que están dispuestas a enfrentar altos niveles de apalancamiento y asumir el riesgo, aunque lo recomendable en materia financiera es mitigar este tipo de necesidades.

Ahora bien, para contrastar la hipótesis inicial, en las siguientes gráficas se puede apreciar la correlación que existe entre el precio del petróleo y el precio de las acciones de las aerolíneas. Esta relación se llevó a cabo con base en la variación relativa diaria del precio del petróleo con referencia WTI y BRENT, y la variación relativa entre días del precio de las acciones. Dichas variaciones ${ }^{11}$ están determinadas por períodos diarios, para un rango de tiempo que oscila entre Enero de 2012 y Enero de 2016.

Las siguientes gráficas están relacionadas con la ecuación 1 y corresponden a la cotización de las seis aerolíneas consideradas en la investigación y el comportamiento de sus acciones con respecto al precio del petróleo con referencia BRENT y WTI.

Gráfica 1: precio de la acción de AEROMEX vs Precio petróleo BRENT

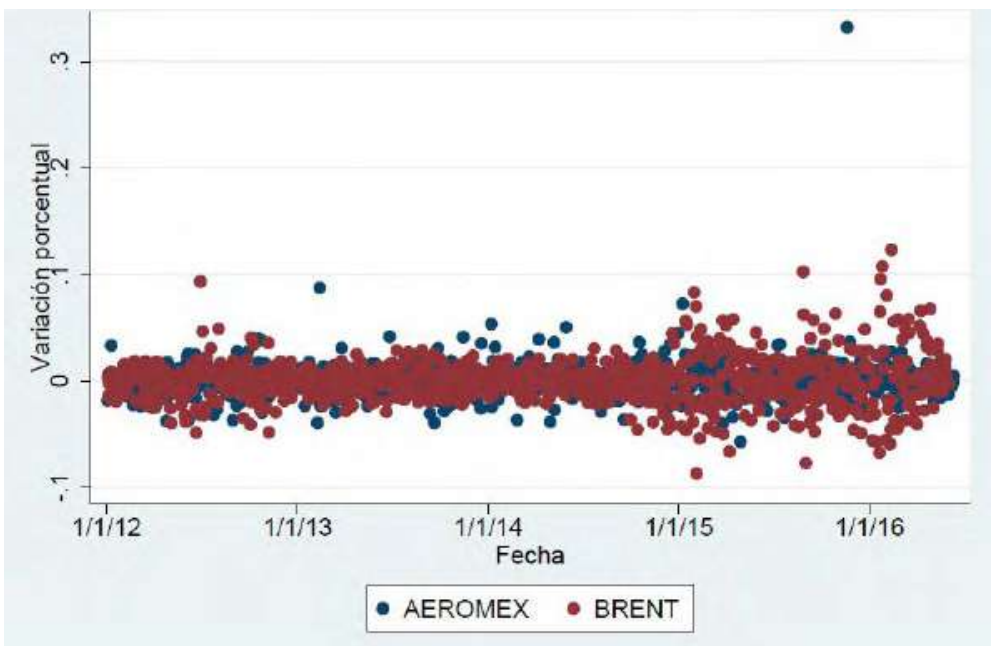

Fuente: elaboración propia a través de modelo en STATA.

11 Las variaciones relativas están determinadas mediante la siguiente fórmula: \%V=Pf-PiPi; donde: $\mathrm{Pf}=$ precio final, $\mathrm{Pi}=$ precio inicial, $\% \mathrm{~V}=$ porcentaje de variación. 
Gráfica 2: precio de la acción de AEROMEX vs Precio petróleo WTI

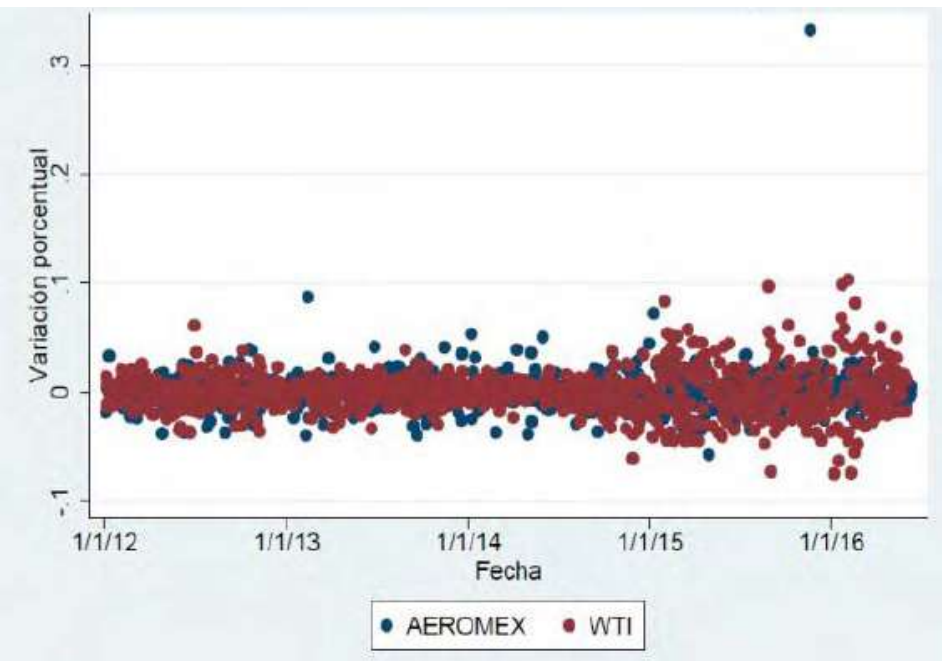

Fuente: elaboración propia a través de modelo en STATA.

Se puede observar que las gráficas 1 y 2 de Aeroméxico son muy parecidas entre sí; hay una relación más fuerte entre los periodos de 2012 a 2015 pues los puntos azules y los puntos rojos que representan los cambios en el precio de las acciones de Aeromex y los precios del petróleo respectivamente se muestran más correlacionados. A partir del 2015 los datos se dispersan un poco y es debido a que las variaciones del precio del petróleo fueron más recurrentes en esta fecha.

Gráfica 3: precio de la acción de AVIANCA vs Precio petróleo BRENT

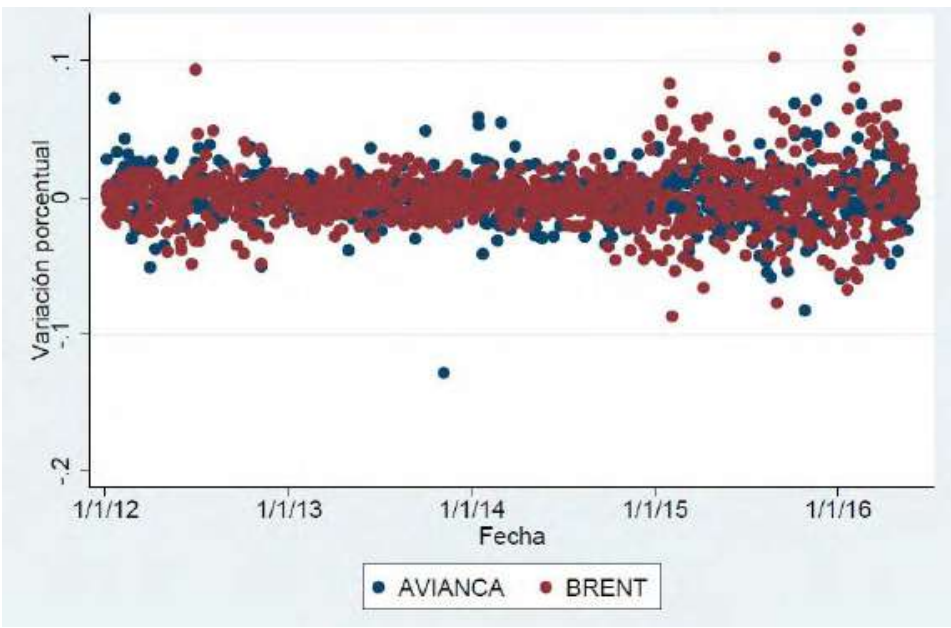

Fuente: elaboración propia a través de modelo en STATA. 
Espinal, L.; Sánchez, M.; Zapata, N. Cotización del petróleo y volumen de acciones de aerolíneas...

Gráfica 4: precio de la acción de AVIANCA vs Precio petróleo WTI

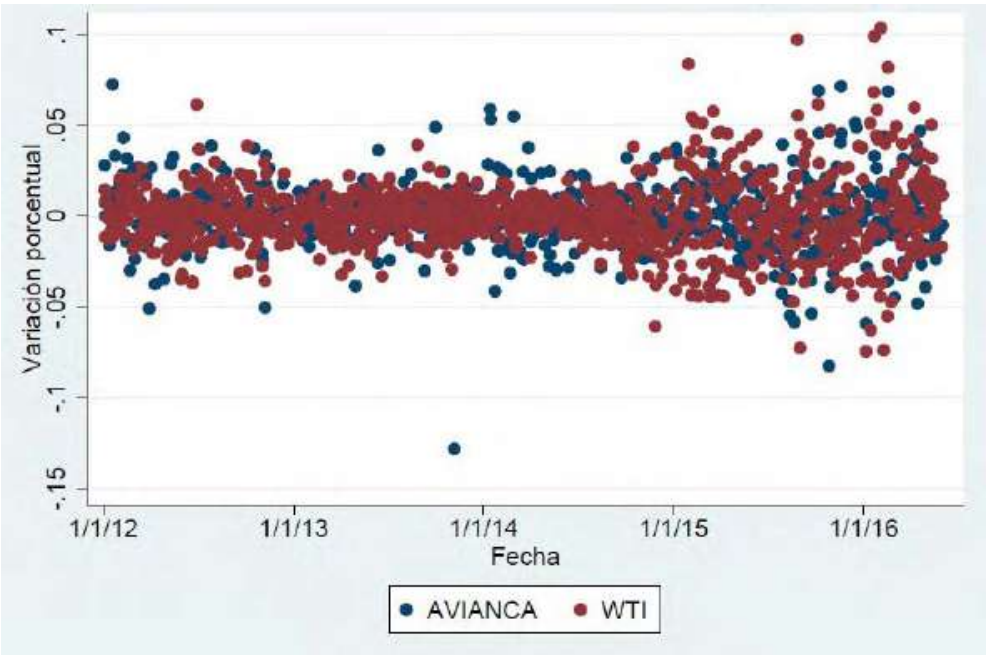

Fuente: elaboración propia a través de modelo en STATA.

Ahora bien, si se observa el comportamiento de la cotización de Avianca en las gráficas 3 y 4, hay una mayor dispersión durante todo el periodo considerado, sin embargo, las variaciones también son más frecuentes a partir de 2015.

Gráfica 5: precio de la acción de COPA vs Precio petróleo BRENT

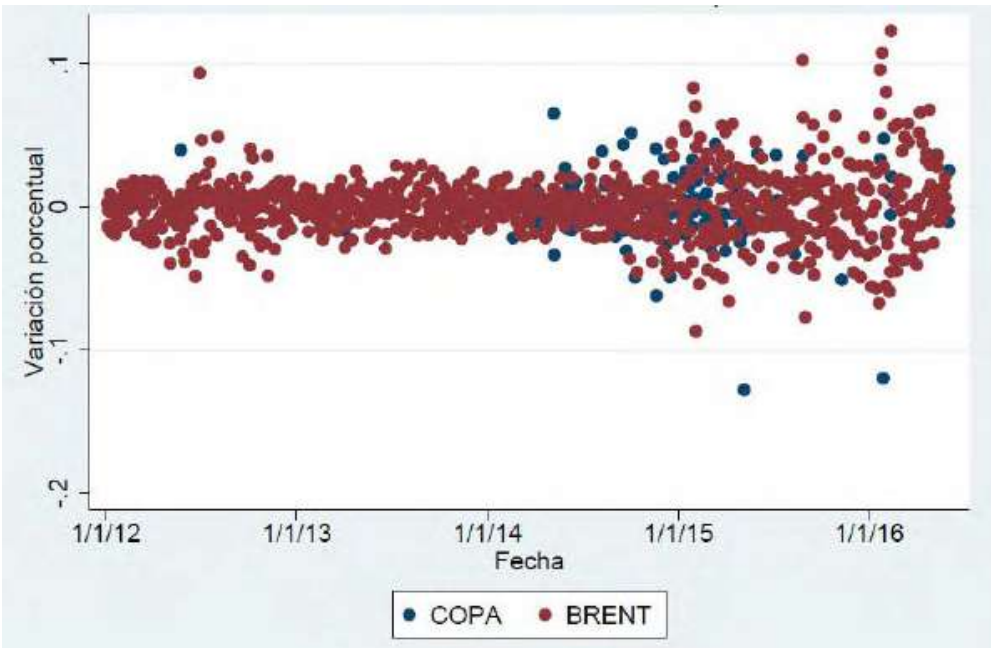

Fuente: elaboración propia a través de modelo en STATA. 
Gráfica 6: precio de la acción de COPA vs Precio petróleo WTI

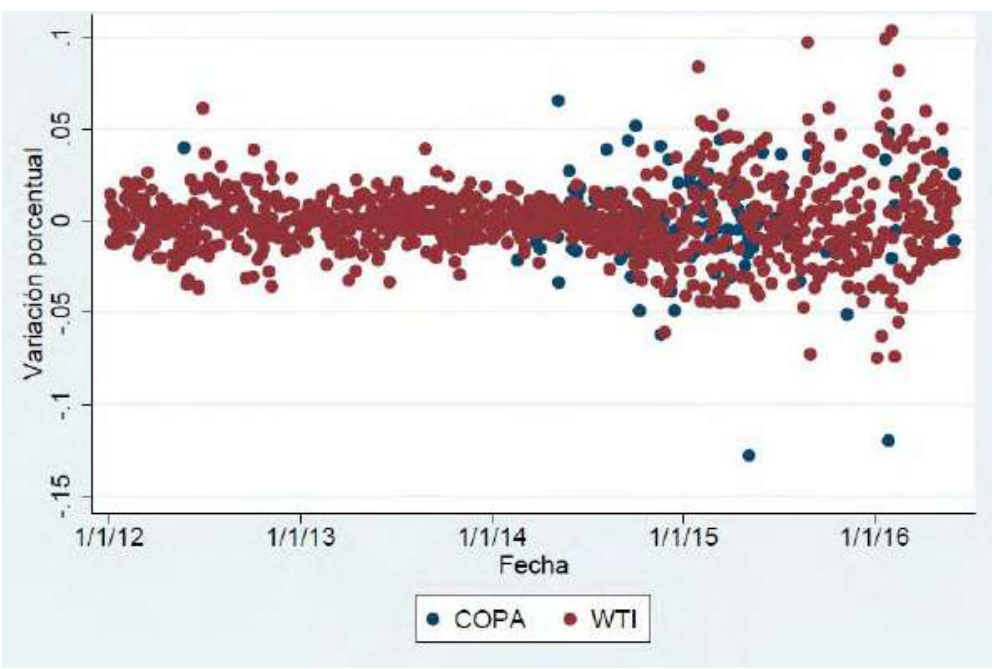

Fuente: elaboración propia a través de modelo en STATA.

Gráfica 7: precio de la acción de VOLARIS vs Precio petróleo BRENT

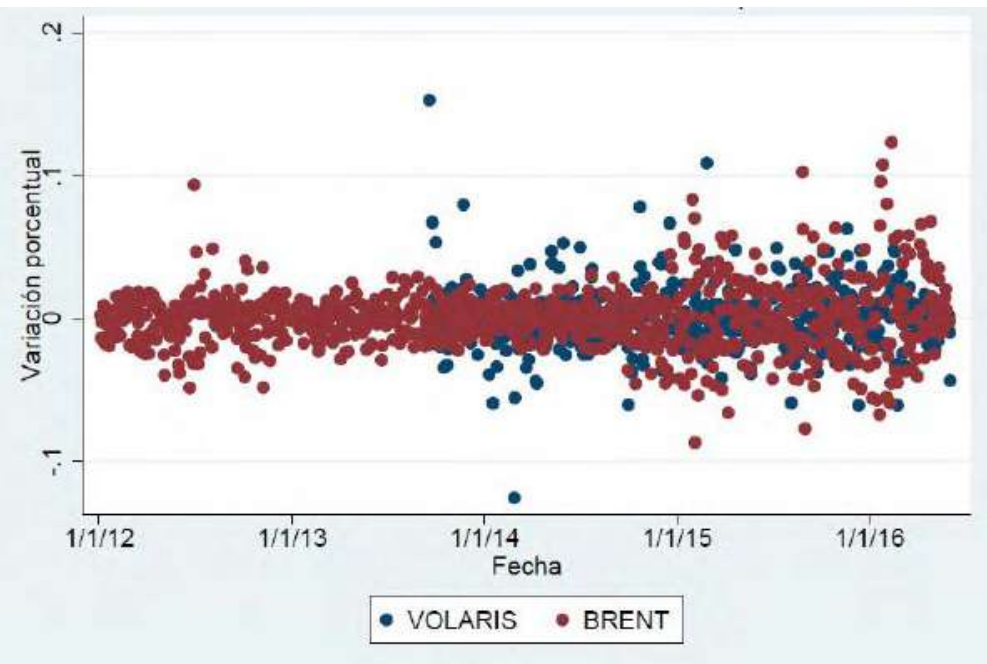

Fuente: elaboración propia a través de modelo en STATA. 
Espinal, L.; Sánchez, M.; Zapata, N. Cotización del petróleo y volumen de acciones de aerolíneas...

Gráfica 8: precio de la acción de VOLARIS vs Precio petróleo WTI

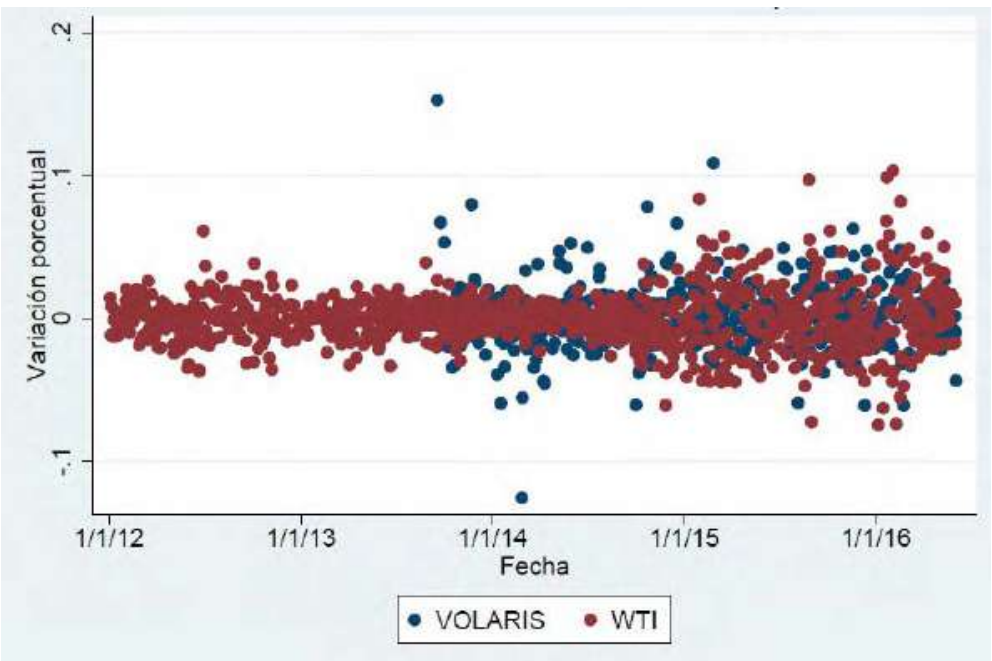

Fuente: elaboración propia a través de modelo en STATA.

Las gráficas 5, 6, 7 y 8, correspondientes a las aerolíneas de Copa y Volaris, estas no muestran en los primeros años de análisis un comportamiento por parte de las acciones ya que por temas estratégicos y de mercado se crean fusiones y absorciones entre las mismas para poder ser más competitivas, tener una porción de mercado mucho más atractiva y ejercer presión en las demás.

Gráfica 9: precio de la acción de GOL vs Precio petróleo BRENT

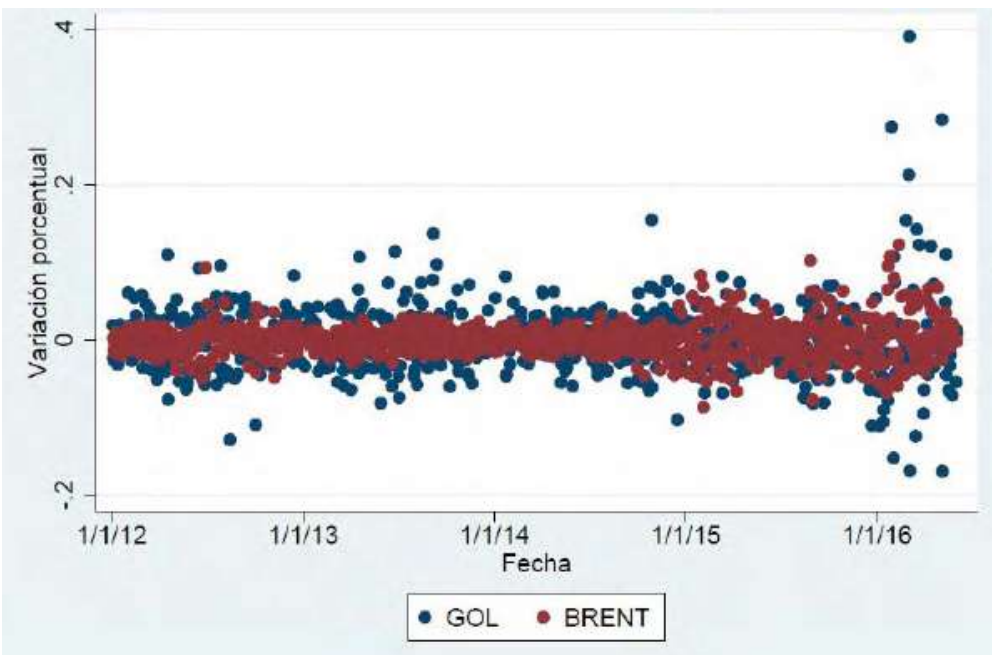

Fuente: elaboración propia a través de modelo en STATA. 
Gráfica 10: precio de la acción de GOL vs Precio petróleo WTI

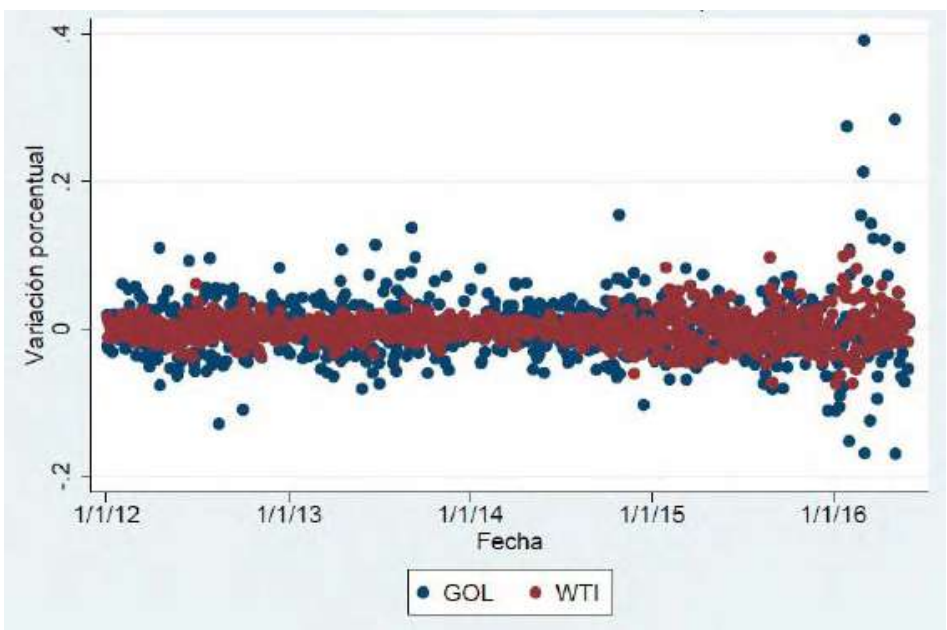

Fuente: elaboración propia a través de modelo en STATA.

Las gráficas 9 y 10, que representan el comportamiento del precio de las acciones de GOL y el precio del petróleo, tiene un comportamiento muy interesante para este análisis ya que hasta 2015 las acciones son más sensibles frente a los cambios del precio del petróleo y su comportamiento constante. Sin embargo, a partir de este mismo año y con la crisis petrolera, al cambiar de manera importante los precios del petróleo, las acciones se comportan de una manera muy correlacionada y enfocada hacia donde migra el petróleo.

Gráfica 11: precio de la acción de LAN vs Precio petróleo BRENT

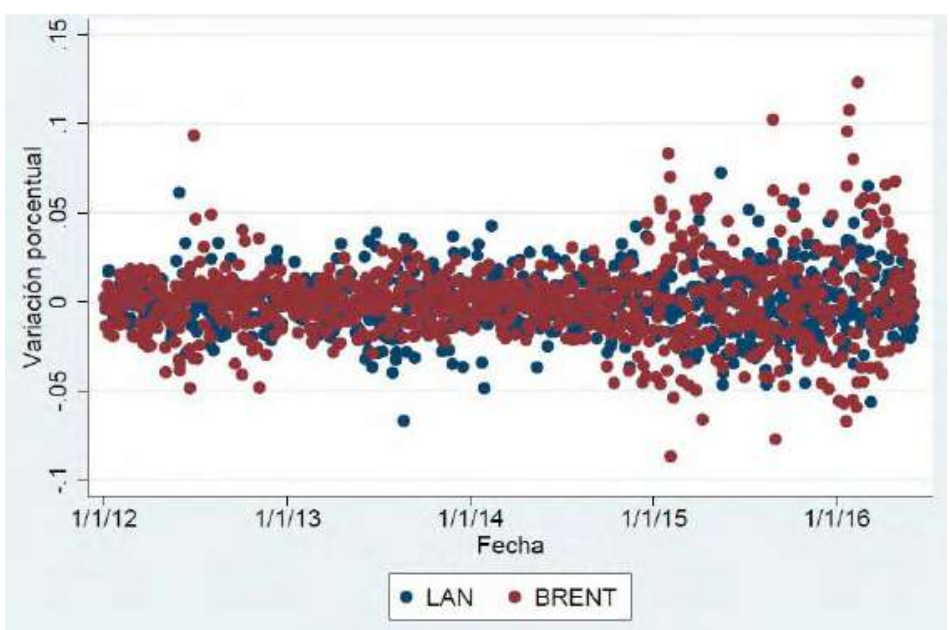

Fuente: elaboración propia a través de modelo en STATA. 
Espinal, L.; Sánchez, M.; Zapata, N. Cotización del petróleo y volumen de acciones de aerolíneas...

Gráfica 12: precio de la acción de LAN vs Precio petróleo WTI

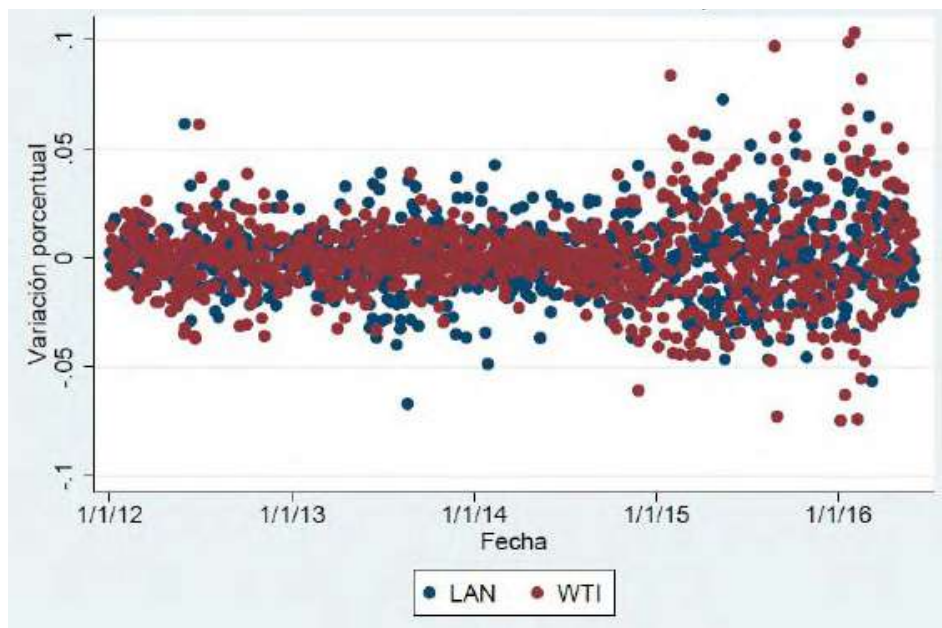

Fuente: elaboración propia a través de modelo en STATA.

Las gráficas 11 y 12 , de las aerolíneas LAN tiene un comportamiento un poco más agresivo frente a las variaciones del petróleo, es decir, hay mucho más movimiento y cambio a partir de 2015 cuando se comienza a percibir con mayor fuerza el impacto petrolero sobre el sector.

En síntesis, un 60\% de las gráficas demuestra la alta sensibilidad del precio de las acciones a la variación del precio del petróleo ya que la zona roja cubre en gran medida la franja azul por sus comportamientos similares.

Se observa que desde el periodo de 2015, donde empieza a evidenciarse una baja en el precio del petróleo, las variaciones diarias del mismo son más drásticas que las variaciones del precio de las acciones, por ello el gráfico de dispersión muestra que los datos se desagregan entre sí.

En la tabla 3 se observa la correlación existente entre el precio de la acción y las dos referencias de petróleo (WTI y BRENT), mediante el coeficiente de correlación (Beta) se muestra la relación entre dichas variables y a través del P-Valor se puede ver si es significativa o no; además se indican el número de observaciones realizadas en el modelo.

Tabla 3. Correlación precio acción vs Precio petróleo

\begin{tabular}{lcccccc}
\hline \multicolumn{1}{c}{ Precio acción } & Volaris & Lan & Gol & Copa & Avianca & Aeromex \\
\hline \multicolumn{1}{c}{ WTI } & & & & & & \\
Coeficiente de correlación & $-0,7306$ & 0,8140 & 0,6446 & 0,3955 & 0,8409 & $-0,6816$ \\
P-Valor & $0,0000^{* * * * * *}$ & $0,0000^{* * * * * *}$ & $0,0000^{* * * *}$ & $0,0000^{* * * * * *}$ & $0,0000^{* * * * *}$ & $0,0000^{* * * * * * *}$ \\
Observaciones & 678 & 1352 & 1336 & 357 & 1218 & 1287
\end{tabular}




\begin{tabular}{|c|c|c|c|c|c|c|}
\hline Precio acción & Volaris & Lan & Gol & Copa & Avianca & Aeromex \\
\hline \multicolumn{7}{|l|}{ BRENT } \\
\hline Coeficiente de correlación & $-0,7354$ & 0,7246 & 0,6463 & 0,4508 & 0,7877 & $-0,7006$ \\
\hline P-Valor & 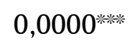 & 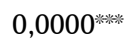 & 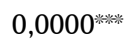 & $0,0000^{\text {米䊉 }}$ & 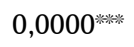 & $0,0000^{\text {橉 }}$ \\
\hline Observaciones & 663 & 1321 & 1308 & 357 & 1191 & 1258 \\
\hline
\end{tabular}

Fuente: elaboración propia a través del modelo STATA.

Se puede afirmar con un nivel de confianza del $99,9 \%$ que la variable del petróleo en sus dos referencias tiene una relación directa con el precio de las acciones, permitiendo así validar la hipótesis planteada inicialmente sobre esta variable.

Es importante señalar que las observaciones realizadas para cada aerolínea varían en tanto no se dispone de información para ciertos años, así, por ejemplo, en el caso de Volaris con respecto a las demás, sólo se realizaron 678 observaciones con respecto a la referencia WTI puesto que apenas empezó a cotizar en bolsa a partir de 2013.

Este modelo permite identificar la correlación existente entre el precio del petróleo y el precio de la acción. Como se observa en la tabla 3, el coeficiente de correlación de todas las empresas es significativo estadísticamente con respecto al WTI y al BRENT, lo que permite corroborar una parte de lo señalado en $\mathrm{H} 1$, además de que las fluctuaciones del precio del petróleo impactan el precio de las acciones de las aerolíneas.

LAN, Gol, Copa y Avianca guardan una correlación positiva con el precio del petróleo, ya que como puede observarse en el signo del coeficiente de correlación de estas empresas con relación al WTI y el BRENT, todas las correlaciones están entre el $40 \%$ y el $84 \%$, lo cual indica que, para dichas aerolíneas, la disminución del petróleo afecta de manera negativa la cotización de su acción.

Por otra parte, la tabla 4, relaciona el volumen de las acciones con el WTI y el BRENT; esta primera variable hace referencia al volumen transado tanto en operaciones de compra y venta.

Tabla 4. Correlación volumen acción vs Precio petróleo

\begin{tabular}{lcccccc}
\hline \multicolumn{1}{c}{ Volumen acción } & Volaris & Lan & Gol & Copa & Avianca & Aeromex \\
\hline \multicolumn{1}{c}{ WTI } & & & & & & \\
Coeficiente de correlación & $-0,2395$ & 0,1021 & $-0,1021$ & $-0,0405$ & $-0,1387$ & 0,0247 \\
P-Valor & $0,0000^{* * * * *}$ & $0,0002^{* * *}$ & $0,0002^{* * * *}$ & 0,4454 & $0,0000^{* * * * * *}$ & 0,3753 \\
Observaciones & 677 & 1350 & 1336 & 357 & 1231 & 1286
\end{tabular}


Espinal, L.; Sánchez, M.; Zapata, N. Cotización del petróleo y volumen de acciones de aerolíneas...

\begin{tabular}{|c|c|c|c|c|c|c|}
\hline Volumen acción & Volaris & Lan & Gol & Copa & Avianca & Aeromex \\
\hline \multicolumn{7}{|l|}{ BRENT } \\
\hline Coeficiente de correlación & $-0,2438$ & 0,0858 & $-0,1337$ & $-0,0452$ & $-0,1161$ & 0,0197 \\
\hline P-Valor & 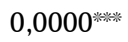 & $0,0018^{\text {粎 }}$ & 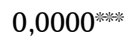 & 0,3944 & $0,0001^{\text {**** }}$ & 0,4863 \\
\hline Observaciones & 662 & 1319 & 1308 & 357 & 1203 & 1257 \\
\hline
\end{tabular}

Fuente: elaboración propia a través del modelo STATA.

En la tabla 4 se muestra que Volaris, LAN, Gol y Avianca presentan un coeficiente de correlación estadísticamente significativo con respecto al WTI de $-23,95 \%, 10,21 \%,-4,05 \%$ y $-13,87 \%$ respectivamente; y con respecto al BRENT una correlación del $-24,38 \%, 8,58 \%,-13,37 \%,-11,61 \%$ respectivamente. Lo anterior indica que a excepción de LAN y Aeroméxico el aumento en el precio del petróleo, tuvo una influencia negativa sobre el volumen de las acciones de las demás compañías.

El volumen de las acciones de LAN presenta una leve correlación positiva frente al precio del petróleo y el volumen de las acciones. Aeroméxico no presenta una correlación estadísticamente significativa que permita inferir que exista una correlación con el precio del petróleo para esta empresa.

Por el contrario, LAN y Aeroméxico guardan una relación positiva con el precio del petróleo, ya que como puede observarse en el signo del coeficiente de correlación de estas empresas con relación al WTI y el BRENT, las relaciones son positivas entre un rango del $2 \%$ y el $10 \%$, lo cual indica que, para dichas aerolíneas, la correlación no es tan alta, el aumento del precio del petróleo no impacta de manera negativa el volumen de transacción de la acción. Es importante señalar que la correlación no es tan alta, pero al analizar el valor p de Aeroméxico se percibe que es significativa en un $37 \%$ y un $48 \%$ en la cotización del WTI y el BRENT, respectivamente.

En conclusión, tras analizar los anteriores resultados, es preciso señalar que la primera hipótesis se cumple, ya que las fluctuaciones del precio del petróleo impactan el precio y volumen de las acciones de las aerolíneas y a su vez afectan su valor económico.

\section{Caracterización de múltiplos comparables de mercado}

Las tablas a continuación muestran el comportamiento de algunos múltiplos que se utilizan para valorar empresas del sector, durante 5 años contados a partir del 2011 para las aerolíneas estudiadas.

La tabla 5 presenta la evolución del PER, Según Álvarez et al, (2006, p, 68) "al valorar empleando el PER no se trata simplemente de obtener el cálculo aritmético del indicador", sin embargo, el alcance de este artículo es el de caracterizar algunos múltiplos. 
Tabla 5. PER aerolíneas

\begin{tabular}{cccccccc}
\hline Año & Aeroméxico & Avianca & Copa & Gol & Lan & Volaris & Media \\
\hline 2011 & 8,92 & 14,66 & 8,31 & 0 & 24,88 & 0 & 11,35 \\
2012 & 10,38 & 11,56 & 13,14 & 0 & 0 & 0 & 7,02 \\
2013 & 11,87 & 6,80 & 16,29 & 0 & 0 & 68,54 & 17,25 \\
2014 & 19,04 & 11,82 & 9,47 & 0 & 0 & 22,14 & 10,41 \\
2015 & 24,10 & 0 & 9,32 & 0 & 0 & 12,16 & 7,60 \\
\hline
\end{tabular}

Fuente: elaboración propia con base en información tomada de Bloomberg.

El PER de la mayoría de aerolíneas en los años 2011 a 2015 oscila entre 6 y 24 veces, diferente al PER de Volaris en el año 2013 el cual asciende a 68,54 veces, dicho comportamiento está fuera de las estadísticas. De igual forma, para los años representados en ceros no se calculó el PER debido a la falta de información.

Asimismo, si en 2013 un comprador quisiera realizar una transacción de compra según el PER, optaría por Volaris ya que reconocería que tiene más capacidad para generar beneficios económicos futuros; ya que, si analiza el siguiente año, también se percibe un mayor beneficio en esta empresa. De igual forma, se observa que, tanto en 2013 como en 2014, el PER de Volaris se encuentra muy por encima de la media del sector.

El siguiente múltiplo a analizar es el precio de mercado sobre el EBITDA, la tabla 6 muestra que, en promedio, el sector tarda entre 4 y 9 veces para regresar el dinero invertido a sus inversores.

Tabla 6. P/EBITDA aerolíneas

\begin{tabular}{cccccccc}
\hline Año & Aeroméxico & Avianca & Copa & Gol & Lan & Volaris & Media \\
\hline 2011 & 3,58 & 3,14 & 5,59 & 19,65 & 8,84 & 0 & 8,16 \\
2012 & 3,19 & 5,12 & 8,91 & $-8,77$ & 11,05 & 0 & 3,90 \\
2013 & 3,05 & 3,45 & 10,79 & 3,19 & 5,16 & 28,99 & 9,11 \\
2014 & 4,46 & 2,96 & 7,17 & 3,90 & 4,14 & 22,10 & 7,45 \\
2015 & 5,18 & 1,18 & 5,05 & 3,01 & 2,07 & 9,26 & 4,29 \\
\hline
\end{tabular}

Fuente: elaboración propia con base en información tomada de Bloomberg.

El P/EBITDA indica cuánto tardaría el flujo de efectivo generado de una empresa en regresarle al inversionista su dinero. En 2015 la empresa más atractiva es Avianca ya que tarda 1,18 veces en retornar el dinero a sus inversores, caso contrario el de Volaris, ya que tarda 9,26 veces. De igual forma, para los años 2013 y 2014, esta última empresa resulta ser la menos atractiva, generando una destrucción de valor con resultado de 28,99 y 22,10 veces por año respectivo. 
Espinal, L.; Sánchez, M.; Zapata, N. Cotización del petróleo y volumen de acciones de aerolíneas...

En la tabla 7, se debe tener en cuenta que el EBIT es un indicador que mide el beneficio operativo de una empresa, no tiene en cuenta ni los intereses ni los impuestos pagados.

Tabla 7. P/EBIT aerolíneas

\begin{tabular}{cccccccc}
\hline Año & Aeroméxico & Avianca & Copa & Gol & Lan & Volaris & Media \\
\hline 2011 & 4,71 & 5,41 & 6,69 & $-12,16$ & 15,76 & 0 & 4,08 \\
2012 & 4,80 & 8,15 & 10,88 & $-3,74$ & 45,66 & 0 & 13,15 \\
2013 & 4,85 & 4,97 & 13,64 & 9,92 & 14,35 & 56,52 & 17,37 \\
2014 & 9,06 & 5,04 & 8,75 & 7,46 & 11,72 & 59,19 & 16,87 \\
2015 & 9,46 & 2,43 & 7,61 & $-4,01$ & 6,38 & 10,95 & 5,47 \\
\hline
\end{tabular}

Fuente: elaboración propia con base en información tomada de Bloomberg.

En ese sentido, en 2011 y 2012, LAN es la empresa que más tarda en retornar su inversión a un potencial inversionista con el flujo operativo y entre 2013 y 2015 la que más tarda es Volaris.

La tabla 8 presenta el EV/EBITDA que compara el valor de una empresa con los beneficios antes de intereses, impuestos, amortizaciones y depreciaciones.

Tabla 8. EV/EBITDA aerolíneas

\begin{tabular}{cccccccc}
\hline Año & Aeroméxico & Avianca & Copa & Gol & Lan & Volaris & Media \\
\hline 2011 & 3,38 & 5,74 & 6,81 & 46,50 & 12,28 & 0 & 14,94 \\
2012 & 3,91 & 8,05 & 10,03 & 0 & 19,47 & 0 & 8,29 \\
2013 & 4,26 & 6,18 & 10,70 & 7,58 & 9,65 & 26,70 & 10,84 \\
2014 & 7,19 & 8,20 & 7,72 & 8,75 & 8,85 & 22,64 & 10,56 \\
2015 & 7,74 & 7,87 & 6,59 & 36,59 & 7,64 & 8,87 & 12,55 \\
\hline
\end{tabular}

Fuente: elaboración propia con base en información tomada de Bloomberg.

Como se observa en la tabla 8, la ratio EV/EBITDA de COPA y LAN sobrepasan la media en el año 2012; GOL está por encima de la media en el año 2011 y 2015; y Volaris la sobrepasa en el 2013 y 2014. Entre menor sea el EV/EBITDA de una empresa se puede suponer que es más eficiente al generar resultados por encima de los recursos invertidos, por lo que resulta más atractiva para un inversionista. Es decir, para los años mencionados, dichas empresas no agregan valor para los inversionistas.

En la tabla 9 se puede observar la ratio que compara el valor de la empresa con sus ventas. 
Tabla 9. EV/Ventas aerolíneas

\begin{tabular}{cccccccc}
\hline Año & Aeroméxico & Avianca & Copa & Gol & Lan & Volaris & Media \\
\hline 2011 & 0,44 & 0,73 & 1,71 & 0,93 & 1,99 & 0 & 1,16 \\
2012 & 0,43 & 0,85 & 2,19 & 0,91 & 2,04 & 0 & 1,29 \\
2013 & 0,45 & 0,74 & 2,68 & 0,70 & 1,21 & 1,27 & 1,18 \\
2014 & 0,56 & 0,84 & 1,81 & 0,84 & 1,12 & 0,88 & 1,01 \\
2015 & 0,91 & 0,81 & 1,17 & 0,90 & 1,08 & 1,45 & 1,05 \\
\hline
\end{tabular}

Fuente: elaboración propia con base en información tomada de Bloomberg.

Este indicador ofrece una idea de cuántas veces el valor de la empresa supera las ventas. En 2011, el valor de LAN supera 1,99 veces sus ventas, valor que está por encima de la media; en 2012 y 2013 Copa vale 2,19 y 2,68 veces sus ventas, respectivamente. Es importante señalar que entre más bajo sea esta ratio la empresa se hace más atractiva, puesto que se espera que la empresa valga más de lo que vale en el presente. Así mismo, se observa que en el periodo 2011, Aeroméxico es la empresa más atractiva a pesar de que LAN vale 1,99 veces sus ventas.

En la tabla 10 se puede observar la relación capitalización bursátil con los recursos propios de la compañía.

Tabla 10. P/VL aerolíneas

\begin{tabular}{cccccccc}
\hline Año & Aeroméxico & Avianca & Copa & Gol & Lan & Volaris & Media \\
\hline 2011 & 2,89 & 1,65 & 1,85 & 1,50 & 5,53 & 0 & 2,68 \\
2012 & 1,90 & 1,95 & 2,85 & 4,86 & 2,20 & 0 & 2,75 \\
2013 & 1,56 & 1,58 & 3,71 & 4,46 & 1,60 & 4,65 & 2,93 \\
2014 & 1,62 & 1,19 & 2,20 & 0 & 1,44 & 3,00 & 1,57 \\
2015 & 2,66 & 0,39 & 1,28 & 0 & 1,00 & 4,38 & 1,62 \\
\hline
\end{tabular}

Fuente: elaboración propia con base en información tomada de Bloomberg.

Cuando las expectativas de generar mayores utilidades aumentan, esta ratio tiende a alejarse de 1, así, durante el periodo comprendido entre 2011 y 2014, las seis aerolíneas aumentaron sus expectativas; GOL, LAN y Volaris son las de mayor ratio. En el 2015, Avianca arrojó un indicador por debajo de 1, esto indica la baja posibilidad de producir excedentes y por tanto una destrucción de valor.

\section{Consideraciones finales}

La desregulación del sector de servicios de transporte aéreo ha aumentado de manera progresiva a través del tiempo por políticas, en su mayoría monetarias, con las cuales se busca impulsar el crecimiento económico de los países, promover el turismo, ser atractivos ante la inversión extranjera y generan beneficios económicos para ayudar al gasto público de los gobiernos. 
Espinal, L.; Sánchez, M.; Zapata, N. Cotización del petróleo y volumen de acciones de aerolíneas...

Las aerolíneas estudiadas en general presentan altos niveles de riesgo de propiedad, puesto que, a medida que hay más participación de terceros en la estructura financiera, estos ganan más poder de negociación y por ende la propiedad de los accionistas se pone en riesgo. Pese a esto Avianca y LAN protagonizan una fuerte presencia en Latinoamérica, en el ámbito financiero y de buen nombre.

Los márgenes EBITDA son aproximados, a excepción de Gol que no llega a la mitad de las demás. Otro factor neurálgico para esta sociedad es que enfrenta un patrimonio negativo, que puede llegar a ser fundamental para su operación.

La industria presenta niveles saludables de efectivo y equivalentes, lo que quiere decir que, cuentan con ventajas financieras, ya que a partir de esto se pueden derivar diversas estrategias que tengan como foco el crecimiento a largo plazo.

Se encontró que LAN y Avianca son las compañías más valoradas y con mejor capital; a excepción de Volaris y Copa, todas las empresas cuentan con un riesgo de propiedad. En especial Gol tiene una situación patrimonial crítica, ya que se encuentra en terreno negativo. Así mismo, se encontró que el aumento del precio del petróleo afecta de forma negativa el precio y volumen de las acciones de las aerolíneas y que los múltiplos cambian de acuerdo a la ratio y a escenarios diferentes al analizado.

Las correlaciones en general indican que el precio del petróleo y el de las acciones de aerolíneas en América Latina es positiva, sin embargo, esto no es lo que se espera según la estructura de costos del sector. Pese a esto, la hipótesis no se cumple en su totalidad en cuanto al precio de las acciones, puesto que apenas dos de las seis empresas estudiadas presentan disminuciones cuando aumenta el precio del petróleo. Sin embargo, cuando se observa el volumen de transacciones, los inversionistas dejan de realizar operaciones cuando la cotización del petróleo aumenta. Un volumen alto indica que existe un interés muy fuerte de una gran cantidad de inversores, mientras que, si el volumen es bajo, hay inversores a la expectativa para tomar una decisión. El hecho de que el volumen de las acciones negociadas guarde una relación negativa con el precio del petróleo es importante, ya que la liquidez de las acciones dependerá también de las fluctuaciones de esta variable.

Por otra parte, al analizar las gráficas en la variable precio de las acciones y precio del petróleo se ve que la relación se hace más evidente, pues en todas las empresas la dispersión es similar. Es decir, frente a posibles cambios en los precios del petróleo en sus dos referencias, los cambios en los precios de las acciones de las aerolíneas se van a comportar con la misma tendencia.

La mayoría de los múltiplos analizados se relacionan con el precio de la acción y el valor de la empresa; en este estudio se evidencia que como mínimo en cada año estudiado, una de las aerolíneas destruyó valor, pues no supera 
los niveles promedios del sector, por ejemplo, al analizar el PER de 2015 se identifica que el de Copa es el más bajo, sin embargo, no destruyó valor en tanto superó la media del sector. Por otra parte, en el mismo año Avianca es la empresa que menos tarda en retornar el capital invertido a los potenciales inversionistas y LAN es la aerolínea más eficiente al generar resultados por encima de los recursos invertidos, pero es más atractiva Avianca al comparar su valor con sus ventas.

El estudio realizado es de alta importancia para los diferentes miembros administrativos de las compañías aeronáuticas evaluadas, al igual que las que no tuvieron alcance en este trabajo, a partir de los mismos se podrán tomar decisiones en cuanto a cobertura de riesgos y la administración de productos derivados, además de esto, podrá servir como base a futuras investigaciones que se deseen realizar en materia de cobertura, valoración de empresas y del sector aeronáutico.

Las debilidades de este estudio se basan principalmente en el número de aerolíneas comprendidas en la investigación, ya que a pesar de que existen gran cantidad de empresas prestadoras de servicios de transporte aéreo en Latinoamérica no son lo suficientemente grandes como para cotizar en bolsa, esto produce que el estudio está centrado únicamente en empresas grandes. Por otra parte, el análisis realizado de la valoración por múltiplos se queda corto ya que no es el objetivo principal del estudio, es importante abrir un análisis amplio de los mismos, pues son una herramienta útil en la valoración.

Como recomendaciones a futuras investigaciones está estudiar la efectividad de las coberturas u otras estrategias encaminadas a disminuir el impacto de la volatilidad del precio del combustible, también, contrastar un análisis del impacto del petróleo con relación al dólar, puntualmente hallar un efecto neto. Analizar la viabilidad de una industria aérea con base en otra fuente energética alternativa para evadir la dependencia del petróleo y por último profundizar en los riesgos de propiedad del sector y el impacto que puedan tener.

\section{Referencias bibliográficas}

Agostini, C. (2008) La organización industrial del transporte aéreo en Chile, En: Revista de Análisis Económico, Vol 23 (1) junio, p. 35-84.

Álvarez, R., García, K., y Borraez, A. (2006). Las razones para valorar una empresa y los métodos empleados. En: Semestre Económico, Vol. 9 (18) julio - diciembre, p. 59-83.

Armstrong, M., y Sappington, D. (2006). Regulation, competition, and liberalization, Journal of Economic Literature. En: Journal of Economic Literatue, Vol. 44 (2), junio, p. 325-366.

ATAG. (2017). Aviation: Benefits without borders, 88p.

Badenes, C., y Santos, J. (1999). Introducción a la valoración de empresas por el método de los múltiplos de compañías comparables. En: IESE Business School, Vol. 299 (34) agosto, p. 1- 24. 
Espinal, L.; Sánchez, M.; Zapata, N. Cotización del petróleo y volumen de acciones de aerolíneas...

Barsky, R., y Kilian, L. (2004). Oil and the macroeconomy since the 1970s, Journal of Economic Perspectives. En: Journal of Economic Perpectives, Vol. 18 (4) p. 115-134.

Bastidas, C. (2007). EBITDA, ¿Es un indicador financiero contable de agregación de valor. En: Capic Review, Vol. 5, p. 41-54.

Bisignani, G. (2006). Airlines, En: Foreing Policy, Vol, 152, p. 22-28.

Blanco, L. (2009). Valoración de empresas por descuento de flujos de caja: Proyección de ratios y estimación del valor terminal por múltiplos. En: Revista Universo Contábil, Vol. 5 (2) abril-junio, p. 125-141.

Blume, L., Easley, D., y O’Hara, M. (1994). Market statistics and technical analysis: The role of volume. En: The Journal of Finance, Vol, 49 (1) marzo, p. 153-181.

Borges, C, A., Yen-Tsang, C., y Maciel, F. (2011). Stock market development : an analysis from a multilevel and multi-country perspective. En: Brazilian Administration Review, Vol. 8 (4) octubre-diciembre, p. 351-375.

Calderón, D. (2012). La importancia y problemática del turbo combustible en la aviación. En: Unimilitar.

Candela, J. (2008). El bajo coste y la nueva aviación comercial. En: Ingeniería Y Territorio, No, 83, p. 80-89.

Cano, G. (2015). Industria de las aerolíneas: La industria y sus riesgos. En: Universidad Pontificia Comillas.

Capoccitti, S., Khare, A., y Mildenberger, U. (2010). Aviation industry - Mitigating climate change impacts through technology and policy. En: Journal of Technology Management \& Innovation, Vol, 5 (2) agosto, p. 66-75.

Cárdenas, C. (2015). El petróleo hoy. En: Economía UNAM, Vol, 12 (35) marzo, p. 100-105.

Correa Mejía, D., Martínez Molina, L., Ruiz Criollo, M., y Yepes Montoya, M. (2018). Los indicadores de costos: una herramienta para gestionar la generación de valor en las empresas industriales colombianas. Estudios Gerenciales, 34(147), 190-199. https:// doi.org/10.18046/j.estger.2018.147.2643

Cuevas, M. (2009). Las condiciones de competencia en las principales rutas de aerolíneas nacionales e internacionales, y los mercados domésticos en cada país del Istmo Centroamericano. En: CEPAL - Serie Estudios Y Perspectivas (120) p, 1-79.

Fernández, P. (2008). Métodos de valoración de empresas. En: IESE Business School, Vol. 3, noviembre.

Fernández, P. (2013). Utilidad y limitaciones de las valoraciones por múltiplos. En: IESE Business School, abril.

Forgas, S., Moliner, M., Sánchez, J, y Palau, R. (2011). La formación de la lealtad de un cliente de una compañía aérea: Diferencias entre aerolíneas tradicionales y de bajo coste, En: Cuadernos de Economia Y Direccion de La Empresa, Vol. 14 (3) julioseptiembre, p, 162-172.

Ginieis, M., Sánchez, M., y Campa, F. (2012). Los costes en el sector del transporte aéreo. En: Universitat Rovira i Virgili, Vol, 10 (20) junio-diciembre, p. 1-21.

Gönenç, R., y Nicoletti, G. (2001). Regulation, market structure and performance in air passenger transportation, En: OECD Economic Studies (32) enero. 
González, A., y Muñoz, C. (2014). Caso de estudio: Easyfly S,A, La primera aerolínea low cost en Colombia, mucho más que una estrategia de precios bajos, En: Universidad EAFIT.

González, J., y Mesa, R. (2007). Los ingresos del petróleo como mecanismo de ajuste externo en Colombia : 2000-2007, En: Perfil de Coyuntura Económica, (9) julio, p. 21-45.

González, S., y Hernández, E. (2016). Impactos indirectos de los precios del petróleo en el crecimiento económico colombiano, En: Lecturas de Economía (84) enero-junio, p. 103-141.

Graham, D., Kaplan, D., y Sibley, D. (1983). Efficiency and competition in the airline industry, Bell Journal of Economics, Vol, 14 (1) p. 118-138.

Gutiérrez, R. (2015). Efectos del choque petrolero de 2014-2015 en la captación de divisas y el gasto público. En: Economía Informa, Vol. 391, maro-abril, p. 54-68.

Gutiérrez, R. (2016). ¿Cómo va minando el entorno petrolero internacional a la economía mexicana? En: Economía Informa, Vol. 397, marzo-abril, p. 80-88.

Helmbold, G. (2004). Crisis de las aerolíneas mundiales, Universidad Nacional del Mar de Plata. En: Universidad Nacional de Mar de Plata, monografía de graduación, 123p.

Hendricks, K. (2005). An Empirical Analysis of the Effect of Supply Chain Disruptions on Long Run Stock Price Performance and Equity Risk of the Firm. Production and Operations, 2p.

Hendricks, K., y Singhal, V. (2003). The effect of supply chain glitches on shareholder wealth. Journal of Operations Management, 21(5), 501-522.

Llinás, M. (2002). Incidencia de la volatilidad de los precios del petróleo en la determinación del ciclo económico colombiano. Tesis para optar al título de magíster en Economía, Maestría en Economía, Universidad de los Andes, Colombia, 66p.

Mateo, J., y García, S., (2014). El sector petrolero en Ecuador, 2000-2010, En: Revista Problemas Del Desarrollo, Vol., 177 (45) abril-junio, p. 113-139.

Meland, W. (2014). Measurement of a cost function for US airlines : restricted and unrestricted translog models, En: Journal of Transport Literature, Vol, 8 (2) abril, p. $38-72$.

Montero, P. (2011). Valoración de empresas, En: Retos, Vol., 1 (1) p. 31-34.

Moreno, D., y Sandoval, O. (2011). Importancia del combustible en el transporte aéreo colombiano, En: Universidad Militar Nueva Granada.

Moscoso, J., y Botero, S. (2013). Métodos de valoración de nuevos emprendimientos. En: Semestre Económico, Vol., 16 (33) junio, p. 237-264.

Narváez, A. (2009). Valoración de empresas: en busca del precio justo, En: Contabilidad Y Negocios, Vol. 4 (7) julio, p. 23-30.

Pagano, M., Panetta, F., y Zingales, L. (1998). Why do companies go public? An empirical analysis. En: The Journal of Finance, Vol. 53 (1) febrero, p. 27-64.

Perilla, J. (2009). Descomposición sectorial y dinámica del impacto de los precios del petróleo sobre el crecimiento económico en Colombia, En: Archivos de Economía, documento 357, julio, 40p. 
Espinal, L.; Sánchez, M.; Zapata, N. Cotización del petróleo y volumen de acciones de aerolíneas...

Perilla, J. (2010). El impacto de los precios del petróleo sobre el crecimiento económico en Colombia, En: Revista de Economía Del Rosario, Vol. 13 (1) p, 76-116.

Realp, J. (2009). Valoración de empresas en el contexto de una "Due diligence": Caso práctico mediante el método de descuento de flujos libres de caja y el método de múltiplos comparables, En: Revista de Contabilidad Y Dirección, Vol. 8, p. 191-206.

Ribeiro, D., y Anson, J. (2007). Análisis de los métodos de valoración utilizados en la práctica: Un estudio con auditores independientes brasileños, En: Revista Universo Contábil, Vol. 3 (3) p. 123-138.

Rojas, E., y Kristjanpoller, W. (2015). Relación precio-volumen mediante análisis de causalidad y efecto día de semana en los mercados accionarios latinoamericanos, En: Lecturas de Economía, Vol. 83, julio-diciembre, p. 9-31.

Santandreu, P., y Torres, J. (2012). Selección del método de valoración de empresas en función de la Empresa y el ciclo económico: El modelo QQC, En: Revista de Contabilidad Y Dirección, Vol. 15, p. 115-132.

Sarmiento, J., y Cayón, E. (2004). Múltiplos para valoración de empresas en Colombia, Análisis de resultados del período 1998-2002, En: Cuadernos de Administración, Vol. 17 (28) julio-diciembre, p. 111-128.

Serrano, J. (2000). Consideraciones críticas en valoración de empresas. En: Academia Revista Latinoamericana de Administración, (24) p. 51-66.

Valdés, V. (2009). Regulación económica de aerolíneas en el Istmo Centroamericano, En: CEPAL - Serie Estudios Y Perspectivas (115) p. 1-71.

Valdés, V., y Ramírez, J. (2011). Una evaluación sobre la desregulación del mercado de aerolíneas en México, En: Economía Mexicana, Nueva Época, Vol. 20 (1) enero, p. $5-35$.

Zapién, B. (2010). Cobertura de la aviación del tipo de cambio y el riesgo precio de combustible en la industria aérea: el caso grupo mexicana de aviación. Tesis para optar al título de Maestro en Ciencias Económicas, Economía Financiera, Instituto Politécnico Nacional, México, 88p. 